\title{
Factorization of Jet Cross Sections in Heavy-Ion Collisions
}

\author{
Jian-Wei Qiu, ${ }^{1, *}$ Felix Ringer, ${ }^{2, \dagger}$ Nobuo Sato, ${ }^{1,3, \$}$ and Pia Zurita ${ }^{4, \S}$ \\ ${ }^{1}$ Theory Center, Jefferson Laboratory, Newport News, Virginia 23606, USA \\ ${ }^{2}$ Nuclear Science Division, Lawrence Berkeley National Laboratory, Berkeley, California 94720, USA \\ ${ }^{3}$ Department of Physics, Old Dominion University, Norfolk, Virginia 23529, USA \\ ${ }^{4}$ Institut für Theoretische Physik, Universität Regensburg, 93040 Regensburg, Germany
}

(Received 12 March 2019; revised manuscript received 13 May 2019; published 24 June 2019)

\begin{abstract}
We propose a new phenomenological approach to establish QCD factorization of jet cross sections in the heavy-ion environment. Starting from a factorization formalism in proton-proton collisions, we introduce medium modified jet functions to capture the leading interaction of jets with the hot and dense QCD medium. A global analysis using a Monte Carlo sampling approach is performed in order to reliably determine the new jet functions from the nuclear modification factor of inclusive jets at the LHC. We find that gluon jets are significantly more suppressed due to the presence of the medium than quark jets. In addition, we observe that the jet radius dependence is directly related to the relative suppression of quark and gluon jets. Our approach may help to improve the extraction of medium properties from data.
\end{abstract}

DOI: $10.1103 /$ PhysRevLett.122.252301

Introduction.-In heavy-ion collisions (HICs) at the LHC and RHIC, hard probes are used to extract information about the created hot and dense QCD medium, the quark-gluon plasma (QGP) [1,2]. Since no parton is observed in isolation, QCD factorization is necessary to separate the physics at different scales and to link the quarks and gluons in hard collisions to the observed hadrons [3]. The factorization has been applied successfully at collider and fixed target experiments. In particular, it is possible to consistently extract universal parton distribution functions (PDFs) within global analyses from different processes and experiments [4-8]. These phenomenological results support the validity of QCD factorization in proton-proton $(p+p)$ collisions and the universality of PDFs, ensuring the predictive power of the approach.

However, QCD factorization in hadron-hadron collisions is an approximation with corrections typically suppressed by inverse powers of the large momentum transfer of the hard scattering. Although the proof of factorization theorems at the leading power of the large momentum transfer is independent of the details of the identified hadrons, the corrections to the factorized formalism are very much sensitive to the hadronic species involved. This is because the subleading power contributions to the hadronic observables are very sensitive to QCD multiple scattering, depending on where the collision is taking place: in a proton, a heavy ion, or a QGP-like hot medium. That is, the

Published by the American Physical Society under the terms of the Creative Commons Attribution 4.0 International license. Further distribution of this work must maintain attribution to the author(s) and the published article's title, journal citation, and DOI. Funded by SCOAP. kinematic regime where the leading power formalism is applicable could be very different for $p+p$, proton-ion, or ion-ion $(A+A)$ collisions. Tremendous efforts have been devoted to study multiple scatterings in QCD, and their medium modifications to hadronic observables from which medium properties were extracted [9-18]. Since only the first subleading power contributions to hadronic observables can be factorized to all orders in perturbative QCD (pQCD) in a similar way as the leading power contributions [19-21], some kind of model dependence is needed for studying QCD multiple scatterings, which can introduce a model bias of the extracted medium properties.

Given the importance of jet quenching observables for extracting QGP properties in HICs, we explore in this Letter the validity of the leading power, model independent QCD factorization formalism for inclusive single jet production in $A+A \rightarrow$ jet $+X$. Using the leading power factorization formalism and the same partonic hard parts and jet evolution for $p+p$ collisions, we demonstrate for the first time that we are able to interpret the jet suppression $R_{A A}^{\mathrm{jet}}$ data from the LHC by fitting medium induced jet functions. We use a Monte Carlo (MC) sampling approach to reliably determine the new medium modified jet functions, and to identify the kinematic regime where the factorization approach is feasible. This data driven approach may open a new door toward extractions of medium properties with a reduced model bias. Eventually, a global analysis of different observables will be needed to establish more rigorously the universality of these nonperturbative functions, and a consistent treatment of medium sensitive power corrections is required to extend the predictive power of our formalism to HICs at lower energies. 
Theoretical framework.-Inclusive single jet cross section in $p+p$ collisions, differential in the transverse momentum $p_{T}$ and rapidity $\eta$, can be factorized as [22]

$$
\begin{aligned}
\frac{d \sigma^{p p \rightarrow \mathrm{jet}+X}}{d p_{T} d \eta} & =\sum_{a b} f_{a / p} \otimes f_{b / p} \otimes \mathcal{H}_{a b}^{\mathrm{jet}} \\
& =\sum_{a b} f_{a / p} \otimes f_{b / p} \otimes\left(\sum_{c} \hat{\sigma}_{a b \rightarrow c} \otimes J_{c}+\hat{\sigma}_{a b}^{\mathrm{jet}}\right) .
\end{aligned}
$$

Here $f_{i / p}\left(x_{i}\right)$ with $i=a, b$ are the PDFs, $\otimes$ indicates appropriate integrals over parton momentum fractions, and $\mathcal{H}_{a b}^{\mathrm{jet}}$ are partonic hard parts for the colliding partons of flavors $a$ and $b$ to produce the observed jet, which are perturbatively calculable depending on the jet algorithm. When the observed jet is very energetic and narrow in cone size $R$, the partonic hard parts $\mathcal{H}_{a b}^{\text {jet }}$ are dominated by large logarithms in $\ln (R)$. Since the $\ln (R)$ are due to the sensitivity to collinear final-state radiation that forms the jet, the resummation of $\alpha_{s}^{n} \ln ^{n}(R)$ is needed, which can be consistently achieved by reorganizing $\mathcal{H}_{a b}^{\mathrm{jet}}$ analogous to Ref. [23]. The separation of $\mathcal{H}_{a b}^{\text {jet }}$ into a "jet-independent" partonic hard part, $\hat{\sigma}_{a b \rightarrow c}(z, \mu)$, for producing a parton $c$ of transverse momentum $p_{T}^{c}=p_{T} / z$ at a factorization scale $\mu \sim p_{T}$ and a "jet-dependent" jet function, $J_{c}\left(z, p_{T} R, \mu\right)$, which accounts for the formation of the observed jet from the parton $c$, as indicated in Eq. (2), allows for the resummation of $\ln (R)$ terms to all orders [24-28]. The $\hat{\sigma}_{a b}^{\text {jet }}$ in Eq. (2) are either $R$ independent or suppressed by powers of $R^{2}$ [29], and they can be neglected if $R$ is sufficiently small. Therefore, we do not consider $\hat{\sigma}_{a b}^{\text {jet }}$ in our analysis. Terms which are further suppressed by inverse powers of $p_{T}$ are also neglected, as they are beyond the factorization formulas in Eqs. (1) and (2).

When $\mathcal{H}_{a b}^{\text {jet }}$ is reorganized for deriving Eq. (2), we can choose the jet-independent $\hat{\sigma}_{a b \rightarrow c}(z, \mu)$ to be the same as the partonic hard part for inclusive single hadron production at high $p_{T}[30,31]$, which is factorized as [32]

$\frac{d \sigma^{p p \rightarrow h+X}}{d p_{T} d \eta}=\sum_{a b c} f_{a / p} \otimes f_{b / p} \otimes \hat{\sigma}_{a b \rightarrow c}(z, \mu) \otimes D_{c}^{h}(z, \mu)$.

Here $D_{c}^{h}$ are the single hadron fragmentation functions (FFs), and the dependence on the initial-state partonic momentum fractions and the factorization scale are left implicit. Since the physically observed cross section on the left-hand side is independent of the factorization scale, the $\mu$ dependence of the FFs follows the Dokshitzer-GribovLipatov-Altarelli-Parisi (DGLAP) evolution, where the evolution kernels are uniquely determined by the $\mu$ dependence of $\hat{\sigma}_{a b \rightarrow c}(z, \mu)$, order by order in pQCD. Since $\hat{\sigma}_{a b \rightarrow c}(z, \mu)$ is the same in both Eqs. (2) and (3), the jet functions obey the same DGLAP evolution equation,

$$
\mu \frac{d}{d \mu} J_{c}\left(z, p_{T} R, \mu\right)=\sum_{d} P_{d c}(z) \otimes J_{d}\left(z, p_{T} R, \mu\right),
$$

with the same $P_{d c}(z)$ as for FFs. Solving the DGLAP evolution equation from the jet invariant mass $\mu_{J} \sim p_{T} R$ to $\mu \sim p_{T}$, the scale of the hard collision, effectively resums single logarithms in the jet radius $\alpha_{s}^{n} \ln ^{n}(R)$. Although the $J_{c}$ in Eq. (2) plays the same role as the $D_{c}^{h}$ in Eq. (3), it is calculable order by order in $\mathrm{pQCD}$, while the FFs are nonperturbative and need to be extracted from the data. The factorized formalism in Eq. (2) has been successfully tested for single inclusive jet production in $p+p$ collisions at the LHC [33].

When we apply Eq. (2) to narrow-cone jet production in HIC, only the PDFs and the jet functions should be modified since $\hat{\sigma}_{a b \rightarrow c}$ is insensitive to the long-distance physics. Although nuclear PDFs (nPDFs) differ from nucleon PDFs, their impact is generally small, consistent with the expectation that jet quenching is a final-state effect [34-37]. That is, the main source of jet quenching is likely to be multiple scattering and medium induced energy loss as the jet traverses the QGP, which modify the $J_{c}$ in $p+p$ collisions into medium sensitive and nonperturbative jet functions $\left(J_{c}^{\mathrm{med}}\right)$,

$$
J_{c}\left(z, p_{T} R, \mu\right) \rightarrow J_{c}^{\operatorname{med}}\left(z, p_{T} R, \mu\right) .
$$

The factorization of jet production in HIC in terms of $J_{c}^{\mathrm{med}}$ was first proposed in Refs. [38,39], where a model calculation [40] was performed. In Ref. [41], the medium modification was taken to be a function of the jet $p_{T}$, and the jet energy loss was determined at the cross section level. Other recent data driven approaches can be found in Refs. [42-44]. The factorization formalism in Eq. (2) with $J_{c}^{\text {med }}$ allows us to directly work at the parton level to study how the parton shower (PS) gets modified due to the presence of the QGP. In Ref. [45], a new approach at the level of jet cross sections was introduced.

We stress that the proposed factorization approach is complementary to others in the literature; see, for example, Ref. [46] and references therein. In-medium calculations based on analytical techniques or PS event generators rely on some kind of factorization in HICs. With the leading power factorization formalism used here, our approach reduces the model bias to a minimum.

To be consistent with QCD factorization at leading power, we leave the DGLAP evolution equation and the corresponding kernels in Eq. (4) unmodified and change only the initial condition of the evolution. In a PS picture this corresponds to keeping the shower between the hard scale $p_{T}$ and the jet scale $p_{T} R$ the same as that in the 
vacuum $[47,48]$. Instead, only the physics at lower scales is affected by the QCD medium, captured effectively by fitting $J_{c}^{\text {med }}$ to the data at the jet scale $\mu_{J} \sim p_{T} R$. This is consistent with the PS developed in Refs. [49-53], where the shower is unmodified relative to the vacuum case at sufficiently large scales. It is possible to extend our calculation to include a medium modified evolution which can be constrained from the data and which we leave for future work [54].

Our analysis here is similar to the global analyses of nPDFs [55-57] and nuclear fragmentation functions in cold nuclear matter [58]. Since the $J_{c}$ are perturbatively calculable, we choose an ansatz where the $J_{c}^{\text {med }}$ are written in terms of the vacuum ones convolved with weight functions $W_{c}(z)$,

$$
J_{c}^{\mathrm{med}}\left(z, p_{T} R, \mu_{J}\right)=W_{c}(z) \otimes J_{c}\left(z, p_{T} R, \mu_{J}\right) .
$$

This approach effectively assumes that the QGP introduces a factorizable modification of the $J_{c}$, which recovers the vacuum case, for example, for very peripheral interactions by having $W_{c}(z) \rightarrow \delta(1-z)$. We adopt the following flexible parametrization,

$$
W_{c}(z)=\epsilon_{c} \delta(1-z)+N_{c} z^{\alpha_{c}}(1-z)^{\beta_{c}},
$$

for the weight functions. As the dependence on the factorization scale $\mu$ of the $J_{c}$ is associated with the leading $\ln (R)$ contribution to the jet cross sections, one finds $\mu(d / d \mu) \int_{0}^{1} d z z J_{c}\left(z, p_{T} R, \mu\right) \propto \sum_{d} \int_{0}^{1} d z z P_{d c}(z)=0$.

That is, the first moment of $J_{c}$ is independent of the factorization scale. Although the number of particles covered by the jet functions in the vacuum and in the medium could be different, and their collinear momenta can be redistributed due to the medium effect, momentum conservation along the direction of the fragmenting parton momentum, $p_{T}^{c}$, requires the $J_{c}$ within the leading power approximation to satisfy the sum rule

$$
\int_{0}^{1} d z z J_{c}\left(z, p_{T}^{c} R, \mu\right)=1
$$

This provides constraints for the evolution of $J_{c}$ in both the vacuum and the medium. The convolution structures in Eqs. (2) and (6) can be handled in Mellin moment space [59]. The parameters of the weight functions are determined by a MC sampling of the likelihood function $\rho(\mathbf{a} \mid$ data $) \propto \mathcal{L}(\mathbf{a}$, data $) \pi(\mathbf{a})$, with $\mathcal{L}(\mathbf{a}$, data $)=\exp \left[-\frac{1}{2} \chi^{2}(\mathbf{a}\right.$, data $\left.)\right]$, where the data resampling method (NNPDF [7] and JAM [8]) is used in order to obtain the MC ensemble for the parameters. The procedure consists of multiple $\chi^{2}$ minimizations, in each of which the data are modified by adding Gaussian noise within the experimentally quoted uncertainties. In addition, we use flat prior parameters with a given range as starting points for each minimization. We have verified that the selected range of the priors does not influence the resulting posterior samples.

Phenomenological results. - We consider inclusive jet data in HICs from the LHC, with the nuclear modification factor defined as

$$
R_{A A}^{\mathrm{jet}}=\frac{d \sigma^{\mathrm{PbPb} \rightarrow \mathrm{jet}+X}}{\left\langle T_{A A}\right\rangle d \sigma^{p p \rightarrow \mathrm{jet}+X}},
$$

where $\left\langle T_{A A}\right\rangle$ is the average nuclear overlap function over a given $A+A$ centrality class [60]. The $J_{c}^{\text {med }}$ need to be extracted separately for different centrality classes and center-of-mass (c.m.) energies. We include all available datasets from the LHC's central collisions $(0 \%-10 \%)$. At $\sqrt{s_{N N}}=2.76 \mathrm{TeV}$, we include the data from ALICE [61], ATLAS [62], and CMS [63], and at $\sqrt{s_{N N}}=5.02 \mathrm{TeV}$, we consider the ATLAS data of Ref. [64] and the preliminary ALICE data of Ref. [65]. For all datasets, the anti- $\mathrm{k}_{T}$ algorithm [66] was used with jet radii in the range of $R=0.2-0.4$. The datasets cover different rapidity ranges, which we take into account without listing here. We add correlated and uncorrelated uncertainties in quadrature. We use the CT14 PDF set of Ref. [5], and we work at next-toleading order, supplemented with resummation at next-toleading logarithmic accuracy. In Fig. 1, we present a comparison of data from the LHC for the $R_{A A}^{\mathrm{jet}}$ with our theoretical results using the fitted $J_{c}^{\text {med }}$. We show the results at $\sqrt{s_{N N}}=2.76 \mathrm{TeV}$ (upper panels) and $\sqrt{s_{N N}}=$ $5.02 \mathrm{TeV}$ (lower panels). For both c.m. energies, we find good agreement with a $\chi^{2} / \mathrm{DOF}$ of $1.1(2.76 \mathrm{TeV})$ and 1.7 $(5.02 \mathrm{TeV})$. At low jet $p_{T}$, there may be an indication for a medium modified DGLAP evolution; however, the precision of current data does not require it yet. More insights

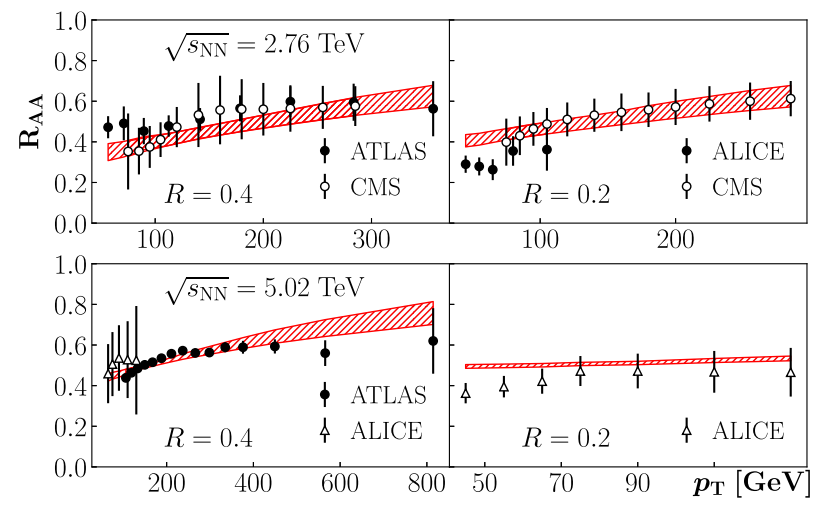

FIG. 1. The $R_{A A}^{\text {jet }}$ for inclusive jet production ( $0 \%-10 \%$ centrality) at (upper panels) $\sqrt{s_{N N}}=2.76 \mathrm{TeV}$ and (lower panels) $\sqrt{s_{N N}}=5.02 \mathrm{TeV}$. We show the comparison with the data from ALICE [61,65], ATLAS [62,64], and CMS [63]. The bands correspond to the full range of $R_{A A}^{\mathrm{jet}}$ values obtained from the MC regression allowed by the data and the parametrization of the medium modification. 


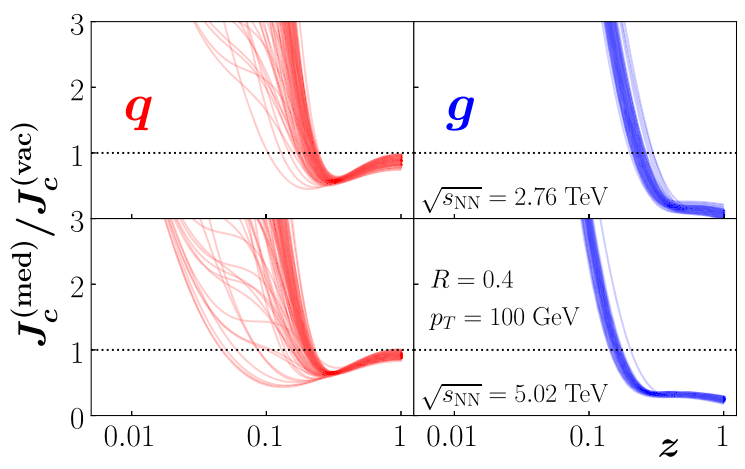

FIG. 2. Ratio of the extracted $J_{c}^{\text {med }}$ and $J_{c}$ at (upper panels) $\sqrt{s_{N N}}=2.76 \mathrm{TeV}$ and (lower panels) $\sqrt{s_{N N}}=5.02 \mathrm{TeV}$ evaluated for $R=0.4$ jets at $\mu=p_{T}=100 \mathrm{GeV}$ for (left panels) quarks and (right panels) gluons.

could be obtained from analyzing hadron and jet substructure observables.

In Fig. 2, we present the ratio of the extracted $J_{c}^{\text {med }}$ and their vacuum analogues for $\sqrt{s_{N N}}=2.76 \mathrm{TeV}$ (upper panels) and $\sqrt{s_{N N}}=5.02 \mathrm{TeV}$ (lower panels) separately for quark (left panels) and gluon (right panels) jets with $R=0.4$ at the scale $\mu=p_{T}=100 \mathrm{GeV}$. The uncertainty at the higher c.m. energy is reduced significantly, mainly due to the very precise dataset from ATLAS at $5.02 \mathrm{TeV}$ [64], which dominates the corresponding fit.

At large $z$, the suppression of the jet functions indicates that it is less likely to form a jet carrying a large momentum fraction of the fragmenting parton in HICs. This is consistent with existing parton energy loss models $[10,12]$. The suppression of $J_{c}^{\text {med }}$ at large $z$ leads to the suppression of the inclusive jet cross section. We note that the HIC jet data put more significant constraints on the large- $z$ region. This is due to the convolution structure of the jet cross section, which forces the phase space with small $x_{a, b}$ and large $z$ to dominate the jet production rate. A possibility to constrain the small- $z$ behavior is the measurement of the energy distribution of inclusive subjets [67].

In Fig. 2, we also observe a significant difference between $J_{q}^{\text {med }}$ and $J_{g}^{\text {med }}$, with gluon jets significantly more suppressed at large $z$ than quark jets. This behavior is expected from model calculations. In fact, we find that it is not possible to fit the experimental data with the same weight function for quarks and gluons in Eq. (7), while retaining a probabilistic interpretation (positivity) of the $J_{c}^{\text {med }}$. We investigated this difference at the level of the cross section, which requires us to define quark and gluon jets beyond leading order. This can be achieved by introducing the jet functions $J_{c d}$ that keep track of the parton $c$ initiating the jet and of the flavor content $d=q, g$ such that $[68,69]$

$$
\sum_{d} J_{c d}\left(z, p_{T} R, \mu\right)=J_{c}\left(z, p_{T} R, \mu\right) .
$$

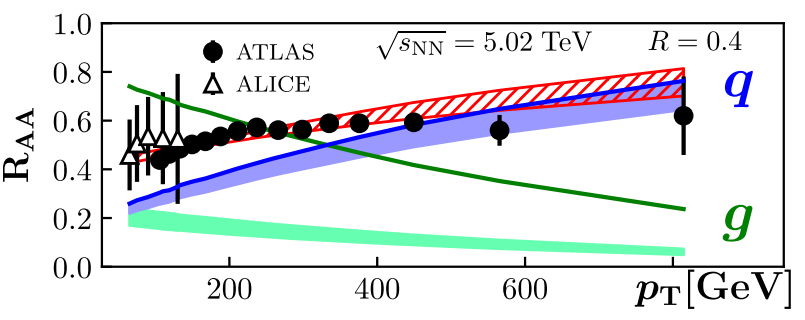

FIG. 3. The suppression of the quark (blue) and gluon (green) cross sections for the lower left panel of Fig. 1 as an example. The individual suppression (bands) can be seen relative to the vacuum fractions (lines). The bands were obtained as in Fig. 1.

In Fig. 3, we show the separation of the vacuum cross section into quark (blue line) and gluon (green line) jets using the $\sqrt{s_{N N}}=5.02 \mathrm{TeV}$ setup (lower left panel of Fig. 1) along with the corresponding separation in the medium (blue and green bands). We observe that gluon jets are significantly more suppressed than quark jets in the medium. Some jet substructure observables support this observation [70-73]. In the future, it will be possible to better pin down differences between quark and gluon jets by including $\gamma / Z+$ jet $[74,75]$ and hadron + jet $[76,77]$ data in a global analysis. We conclude that the leading power factorization formalism with medium jet functions not only captures the feature of in-medium interactions of jets with the QGP but also allows for a clear physical interpretation.

An intriguing aspect of jet quenching studies is the jet radius dependence. While the current data remains inconclusive, different model calculations predict the $R_{A A}^{\mathrm{jet}}$ to either increase or decrease with $R$. In general, a nonmonotonic behavior is expected: the $R_{A A}^{\mathrm{jet}}$ increases at both formal limits $R \rightarrow 0, \infty$. In the limit $R \rightarrow 0$, the $R_{A A}^{\mathrm{jet}}$ is expected to approach the hadron $R_{A A}^{h}$, which is generally above the $R_{A A}^{\mathrm{jet}}$ [78]. For large $R$, the energy lost by partons due to medium interactions should eventually all be contained in a very large cone. However, both limits are formally not covered by the factorization formalism in Eq. (2). For $R \rightarrow 0$, the jet scale $\mu_{J} \sim p_{T} R \rightarrow 0$, and the evolution starts at $\mu_{J} \sim 1 \mathrm{GeV}$ with a nonperturbative $J_{c}$. For the experimentally accessible $R$ values it is a priori not clear whether the $R_{A A}^{\mathrm{jet}}$ increases or decreases with $R$. In Fig. 4, we show the $R$ dependence obtained within our framework at $\sqrt{s_{N N}}=5.02 \mathrm{TeV}$. In the vacuum, the gluon fraction of the jets decreases with smaller $R$, caused by more phase space to evolve and the $J_{g}$ evolving faster, leading to the increase of the quark fraction (lower right panel). In the medium, gluon jets are more significantly quenched (lower left panel), which is why the $R_{A A}^{\text {jet }}$ (upper panel) effectively inherits the $R$ dependence of the quark jets. It will be interesting to see whether these findings will be confirmed by more precise data in the future. 


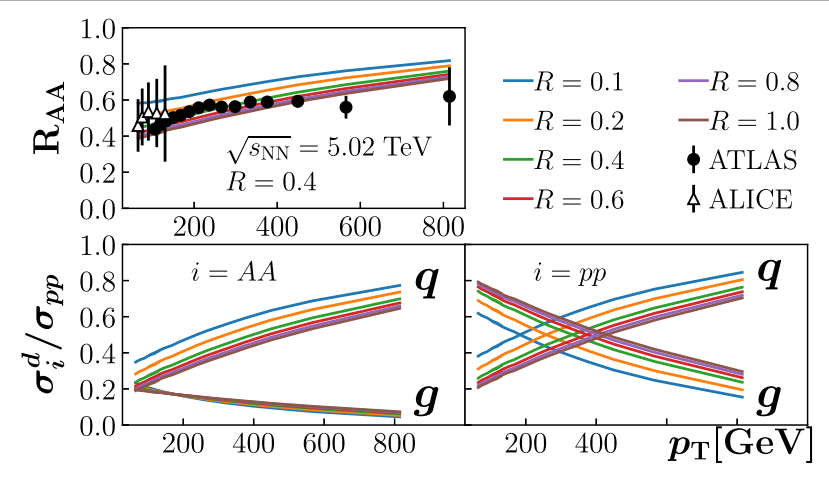

FIG. 4. The dependence of the $R_{A A}^{\mathrm{jet}}$ at $\sqrt{s_{N N}}=5.02 \mathrm{TeV}$ on (upper panel) the jet radius $R$, and quark and gluon jet contributions $\sigma_{i}^{d} / \sigma_{p p}$ with $d=q, g$-see Eq. (10)-in (lower left panel) the medium $i=A A$ and (lower right panel) vacuum $i=p p$.

Conclusions.-In this Letter, we proposed an approach to phenomenologically establish QCD factorization of jet cross sections in HIC. We considered inclusive jet production at the LHC and found that it is possible to describe the $R_{A A}^{\mathrm{jet}}$ by the leading power factorization formalism for $p+p$ collisions with medium modified jet functions. Our results support the notion of QCD factorization in the HIC environment. In our framework, it is possible to separate quark and gluon jets. We found that gluon jets are significantly more suppressed than quark jets, and there is a direct link between the relative suppression of quark and gluon jets and the jet radius dependence of the $R_{A A}^{\text {jet }}$.

It is critically important to investigate the universality of the jet functions to ensure the predictive power. We plan to analyze $\gamma / Z$ tagged jet data as well as hadron and jet substructure observables in a similar way. The intuitive physical interpretation of the extracted medium jet functions may facilitate comparisons with model calculations. Our proposed approach helps identify the impact of the medium modification at the parton level, and it may serve as guidance for constructing microscopic models of the QGP and its interaction with hard probes. We hope that the factorization framework may help to explore how the formation and the evolution of a parton shower gets modified due to the presence of the hot and dense QCD medium created in HICs, from which the properties of the QGP can be better extracted.

We thank Y.-T. Chien, R. Elayavalli, Z.-B. Kang, K. Lee, Y.-J. Lee, A. Majumder, Y. Mehtar-Tani, M. Ploskon, A. Sickles, M. Spousta, I. Vitev, W. Vogelsang, W. Waalewijn, and F. Yuan for the very helpful discussions, and C. Andres, F. Dominguez, P. Jacobs, J. Mulligan and X.-N. Wang also for valuable suggestions about the manuscript. This work was supported in part by U.S. Department of Energy under Contracts No. DE-AC05-06OR23177, No. DE-AC0205CH11231, and No. DE-SC0012704, and the LDRD program at LBNL. *jqiu@jlab.org

fmringer@lbl.gov

*nsato@jlab.org

§maria.zurita@ur.de

[1] M. Gyulassy, I. Vitev, X.-N. Wang, and B.-W. Zhang, in Quark-Gluon Plasma 3, edited by R. C. Hwa and X.-N. Wiang (World Scientific, Singapore, 2004), pp. 123-191.

[2] A. Accardi et al., arXiv:hep-ph/0310274.

[3] J. C. Collins, D. E. Soper, and G. F. Sterman, Adv. Ser. Dir. High Energy Phys. 5, 1 (1989).

[4] L. A. Harland-Lang, A. D. Martin, and R. S. Thorne, Eur. Phys. J. C 78, 248 (2018).

[5] S. Dulat, T.-J. Hou, J. Gao, M. Guzzi, J. Huston, P. Nadolsky, J. Pumplin, C. Schmidt, D. Stump, and C.-P. Yuan, Phys. Rev. D 93, 033006 (2016).

[6] S. Alekhin, J. Blümlein, S. Moch, and R. Placakyte, Phys. Rev. D 96, 014011 (2017).

[7] R. D. Ball et al. (NNPDF Collaboration), Eur. Phys. J. C 77, 663 (2017).

[8] A. Accardi, L. T. Brady, W. Melnitchouk, J. F. Owens, and N. Sato, Phys. Rev. D 93, 114017 (2016).

[9] M. Gyulassy and X.-n. Wang, Nucl. Phys. B420, 583 (1994).

[10] R. Baier, Y. L. Dokshitzer, A. H. Mueller, S. Peigne, and D. Schiff, Nucl. Phys. B484, 265 (1997).

[11] B. G. Zakharov, JETP Lett. 63, 952 (1996).

[12] M. Gyulassy, P. Levai, and I. Vitev, Nucl. Phys. B594, 371 (2001).

[13] X.-N. Wang and X.-f. Guo, Nucl. Phys. A696, 788 (2001).

[14] P. B. Arnold, G. D. Moore, and L. G. Yaffe, J. High Energy Phys. 06 (2002) 030.

[15] J.-W. Qiu and I. Vitev, Phys. Lett. B 632, 507 (2006).

[16] N. Armesto, A. Buzzatti, N. Chang, C. Gale, M. Gyulassy, U. Heinz, S. Jeon, A. Majumder, B. Müller, G.-Y. Qin et al., Phys. Rev. C 86, 064904 (2012).

[17] K. M. Burke et al. (JET Collaboration), Phys. Rev. C 90, 014909 (2014).

[18] C. Andres, N. Armesto, H. Niemi, R. Paatelainen, and C. A. Salgado, arXiv:1902.03231.

[19] J.-W. Qiu and G. F. Sterman, Nucl. Phys. B353, 137 (1991).

[20] J. Botts, J.-W. Qiu, and G. F. Sterman, Nucl. Phys. A527, 577 (1991).

[21] J.-W. Qiu, arXiv:hep-ph/0305161.

[22] S. D. Ellis and D. E. Soper, Phys. Rev. D 48, 3160 (1993).

[23] E. L. Berger, J.-W. Qiu, and X.-f. Zhang, Phys. Rev. D 65, 034006 (2002).

[24] M. Dasgupta, F. Dreyer, G. P. Salam, and G. Soyez, J. High Energy Phys. 04 (2015) 039.

[25] T. Kaufmann, A. Mukherjee, and W. Vogelsang, Phys. Rev. D 92, 054015 (2015).

[26] Z.-B. Kang, F. Ringer, and I. Vitev, J. High Energy Phys. 10 (2016) 125.

[27] L. Dai, C. Kim, and A. K. Leibovich, Phys. Rev. D 94, 114023 (2016).

[28] L. Dai, C. Kim, and A. K. Leibovich, J. High Energy Phys. 09 (2018) 109.

[29] S. D. Ellis, Z. Kunszt, and D. E. Soper, Phys. Rev. Lett. 69, 3615 (1992).

[30] F. Aversa, P. Chiappetta, M. Greco, and J. P. Guillet, Nucl. Phys. B327, 105 (1989). 
[31] B. Jager, A. Schafer, M. Stratmann, and W. Vogelsang, Phys. Rev. D 67, 054005 (2003).

[32] G. C. Nayak, J.-W. Qiu, and G. F. Sterman, Phys. Rev. D 72, 114012 (2005).

[33] X. Liu, S.-O. Moch, and F. Ringer, Phys. Rev. D 97, 056026 (2018).

[34] J. Adams et al. (STAR Collaboration), Phys. Rev. Lett. 91, 072304 (2003).

[35] S. S. Adler et al. (PHENIX Collaboration), Phys. Rev. Lett. 91, 072303 (2003).

[36] V. Khachatryan et al. (CMS Collaboration), Eur. Phys. J. C 76, 372 (2016).

[37] S. Acharya et al. (ALICE Collaboration), Phys. Lett. B 783, 95 (2018).

[38] Z.-B. Kang, F. Ringer, and I. Vitev, Phys. Lett. B 769, 242 (2017).

[39] H. T. Li and I. Vitev, arXiv:1811.07905.

[40] G. Ovanesyan and I. Vitev, J. High Energy Phys. 06 (2011) 080.

[41] Y. He, L.-G. Pang, and X.-N. Wang, arXiv:1808.05310.

[42] J. Casalderrey-Solana, Z. Hulcher, G. Milhano, D. Pablos, and K. Rajagopal, Phys. Rev. C 99, 051901 (2019).

[43] W. Ke, Y. Xu, and S. A. Bass, Phys. Rev. C 98, 064901 (2018).

[44] C. Sirimanna, S. Cao, and A. Majumder, Proc. Sci. HardProbes2018 (2019) 053 [arXiv:1901.03635].

[45] J. Brewer, J. G. Milhano, and J. Thaler, arXiv:1812.05111 [Phys. Rev. Lett. (to be published)].

[46] X.-N. Wang, Quark-Gluon Plasma 5 (World Scientific, Singapore, 2016).

[47] J. Casalderrey-Solana, Y. Mehtar-Tani, C. A. Salgado, and K. Tywoniuk, Phys. Lett. B 725, 357 (2013).

[48] P. Caucal, E. Iancu, A. H. Mueller, and G. Soyez, Phys. Rev. Lett. 120, 232001 (2018).

[49] K. Zapp, G. Ingelman, J. Rathsman, J. Stachel, and U. A. Wiedemann, Eur. Phys. J. C 60, 617 (2009).

[50] B. Schenke, C. Gale, and S. Jeon, Phys. Rev. C 80, 054913 (2009).

[51] X.-N. Wang and Y. Zhu, Phys. Rev. Lett. 111, 062301 (2013).

[52] S. Cao and A. Majumder, arXiv:1712.10055.

[53] S. Cao, C. Park, R. A. Barbieri, S. A. Bass, D. Bazow, J. Bernhard, J. Coleman, R. Fries, C. Gale, Y. He et al. (JETSCAPE Collaboration), Phys. Rev. C 96, 024909 (2017).

[54] N. Armesto, L. Cunqueiro, and C. A. Salgado, Eur. Phys. J. C 63, 679 (2009).
[55] K. J. Eskola, P. Paakkinen, H. Paukkunen, and C. A. Salgado, Eur. Phys. J. C 77, 163 (2017).

[56] D. de Florian, R. Sassot, P. Zurita, and M. Stratmann, Phys. Rev. D 85, 074028 (2012).

[57] K. Kovarik et al., Phys. Rev. D 93, 085037 (2016).

[58] R. Sassot, M. Stratmann, and P. Zurita, Phys. Rev. D 81, 054001 (2010).

[59] D. de Florian, R. Sassot, M. Stratmann, and W. Vogelsang, Phys. Rev. D 80, 034030 (2009).

[60] D. G. d'Enterria, arXiv:nucl-ex/0302016.

[61] J. Adam et al. (ALICE Collaboration), Phys. Lett. B 746, 1 (2015).

[62] G. Aad et al. (ALICE Collaboration), Phys. Lett. B 719, 220 (2013).

[63] V. Khachatryan et al. (CMS Collaboration), Phys. Rev. C 96, 015202 (2017).

[64] M. Aaboud et al. (ALICE Collaboration), Phys. Lett. B 790, 108 (2019).

[65] J. Mulligan (ALICE Collaboration), Proc. Sci. HardProbes2018 (2019) 080 [arXiv:1812.07681].

[66] M. Cacciari, G. P. Salam, and G. Soyez, J. High Energy Phys. 04 (2008) 063.

[67] Z.-B. Kang, F. Ringer, and W. J. Waalewijn, J. High Energy Phys. 07 (2017) 064.

[68] A. Banfi, G. P. Salam, and G. Zanderighi, Eur. Phys. J. C 47, 113 (2006).

[69] P. Cal, F. Ringer, and W. J. Waalewijn, J. High Energy Phys. 05 (2019) 143.

[70] S. Acharya et al. (ALICE Collaboration), J. High Energy Phys. 10 (2018) 139.

[71] M. Aaboud et al. (ALICE Collaboration), Phys. Rev. C 98, 024908 (2018).

[72] A. M. Sirunyan et al. (CMS Collaboration), Phys. Rev. Lett. 121, 242301 (2018).

[73] M. Spousta and B. Cole, Eur. Phys. J. C 76, 50 (2016).

[74] A. M. Sirunyan et al. (CMS Collaboration), Phys. Lett. B 785, 14 (2018).

[75] M. Aaboud et al. (ATLAS Collaboration), Phys. Lett. B 789, 167 (2019).

[76] J. Adam et al. (ALICE Collaboration), J. High Energy Phys. 09 (2015) 170.

[77] L. Adamczyk et al. (STAR Collaboration), Phys. Rev. C 96, 024905 (2017).

[78] V. Khachatryan et al. (CMS Collaboration), J. High Energy Phys. 04 (2017) 039. 